\title{
Impact of molecular absorption on the design of free space optical communications 10502018
}

G. Artaud (a), B. Benammar (a), D. Jouglet (a), L. Canuet (a), J. Lacan (b)

(a) Centre National d'Etudes Spatiales (French Space Agency), Toulouse France;

(b) Dept. of Complex Systems Engineering - ISAE-SUPAERO, University of Toulouse

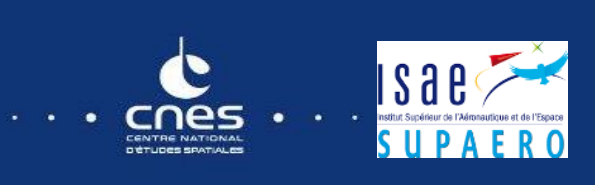




\section{Outline}

Satellite optical communications Impact of absorption on PAT beacon Impact of absorption on data WDM Conclusion 


\section{Satellite optical communications}

Optical links through the atmosphere

- LEO direct to Earth

- GEO feeder links

- LEO MEO constellation feeder links

- GEO relay (ISL)

Propagation challenges

- Absorption (molecules)

- Scattering (aerosols),

- Cloud blockage

- Atmospheric turbulence

Envisaged wavelength plan

- C band [1530-1565 nm] and L band [1565-1625 nm]

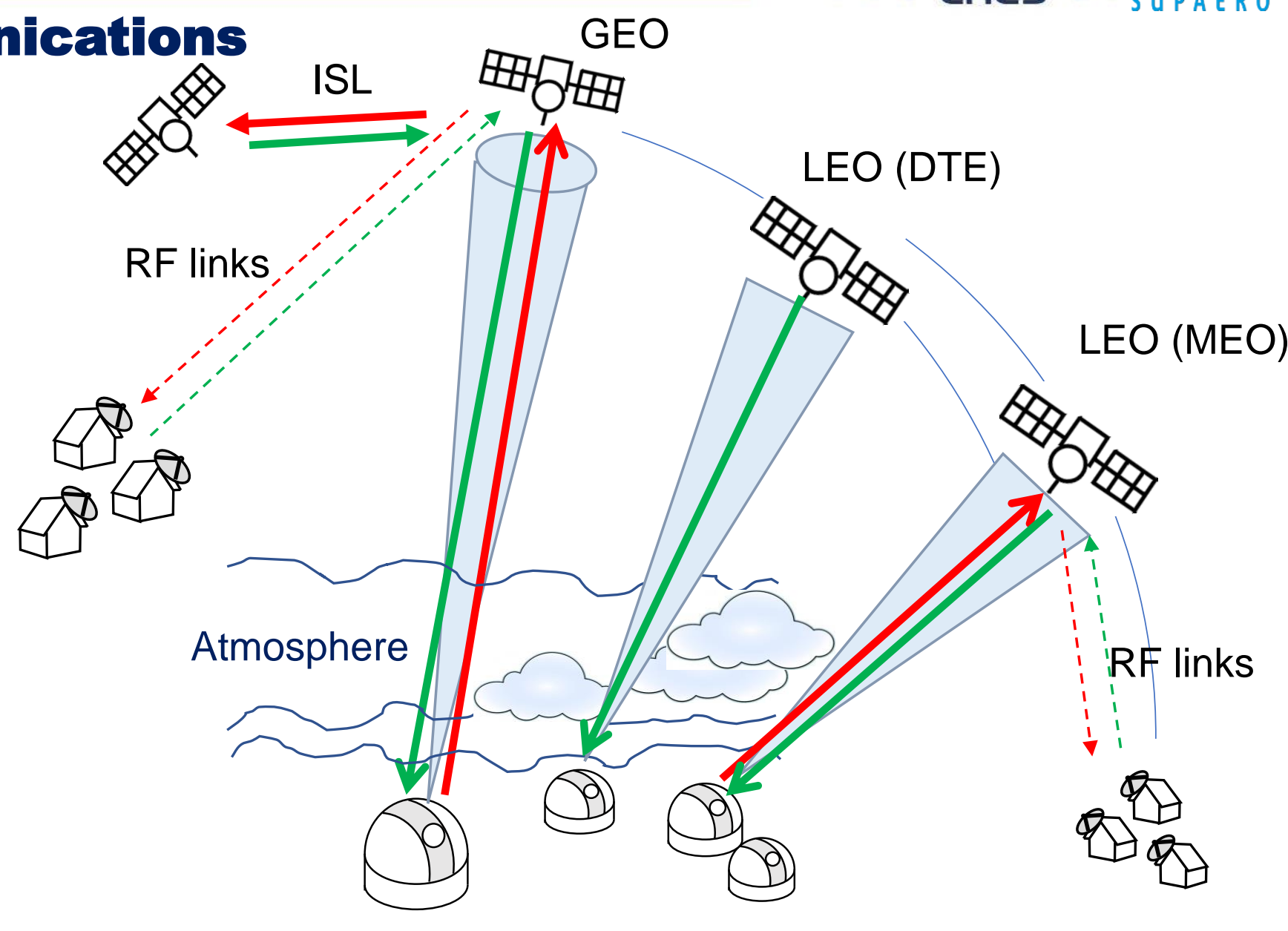




\section{Atmospheric absorption}

\section{Total atmopheric absorption depends on:}

- Types and concentration of molecules

- Thickness of the atmosphere (elevation angle)

- Climatic region (H2O)

\section{Simulation of molecular transmission}

- $4 \mathrm{~A} / \mathrm{OP}$ Radiative transfer tool (CNES, LMD Noveltis)

- High resolution Line by Line transmission simulation in the infra-red region

$$
0 \leq \text { Transmission } \leq 1
$$

$$
\text { Attenuation }[\mathrm{dB}]=-10 \log _{10} \text { (transmission) }
$$

- 5 climatic regions (tropical, mid lat summer, mid lat winter, surb arc summer and sub arc winter)

- 4 elevation angles $\left(5^{\circ}, 10^{\circ}, 20^{\circ}, 40^{\circ}\right)$

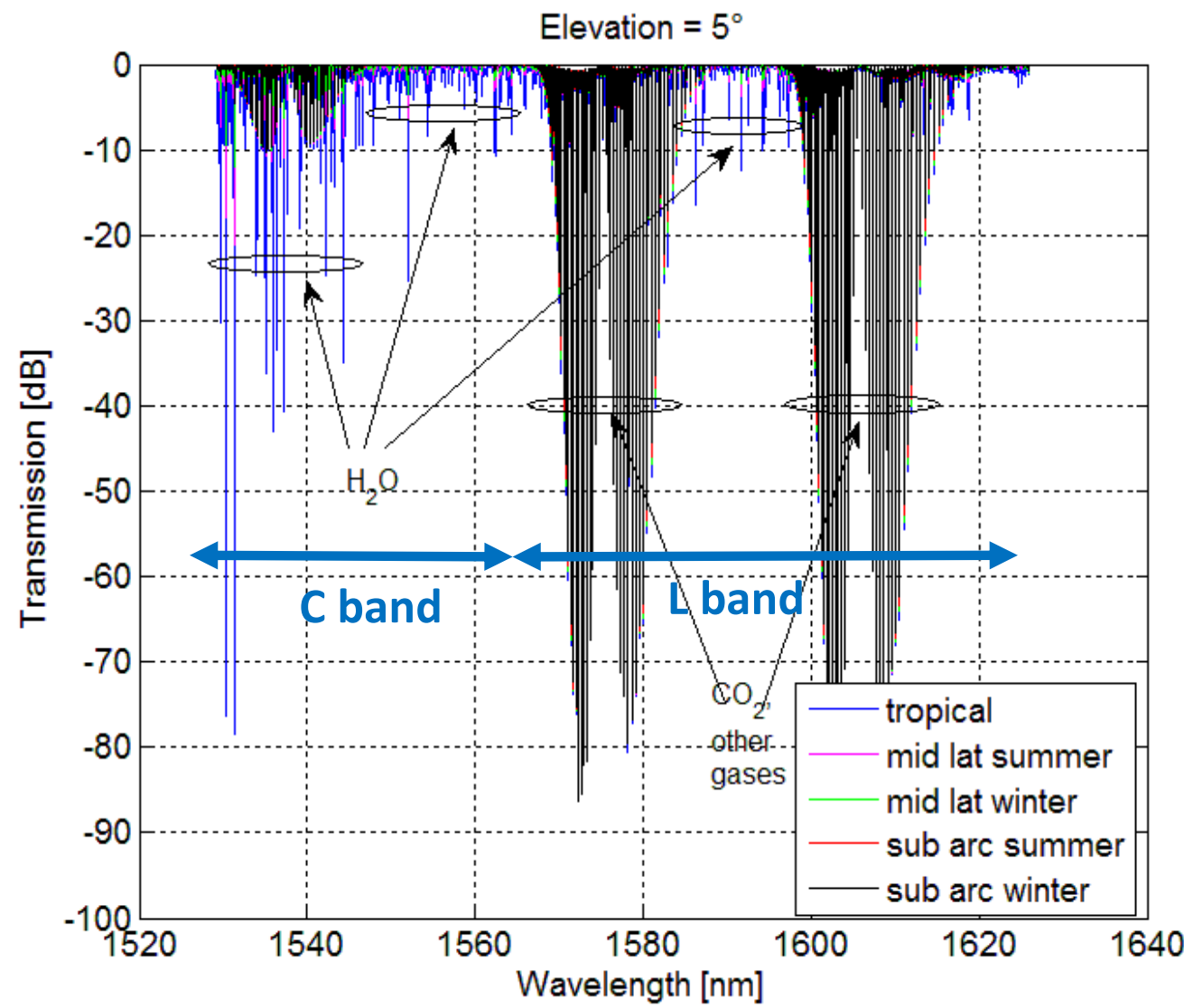




\section{Atmospheric absorption : climatic dependence}
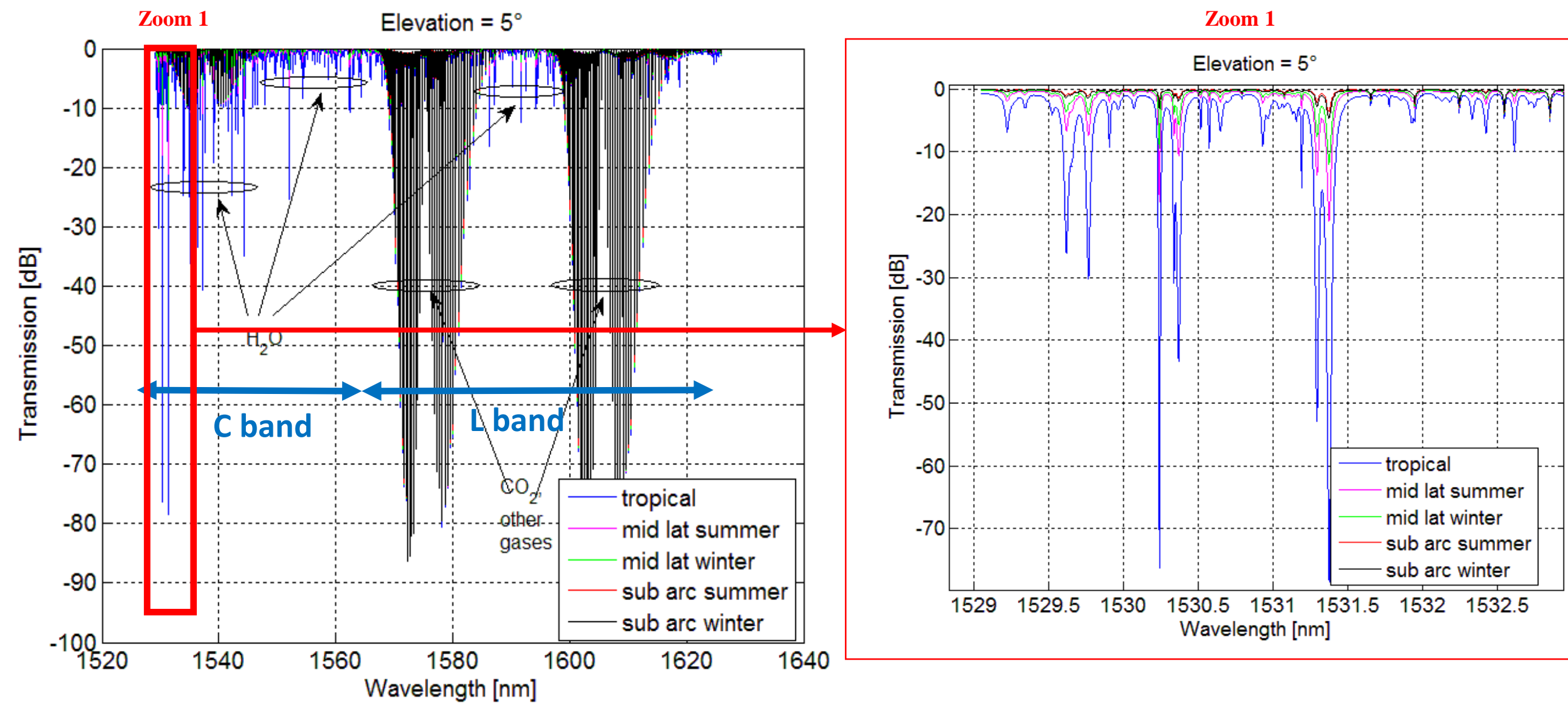


\section{Atmospheric absorption : climatic dependence}

Zoom 2

Elevation $=5^{\circ}$

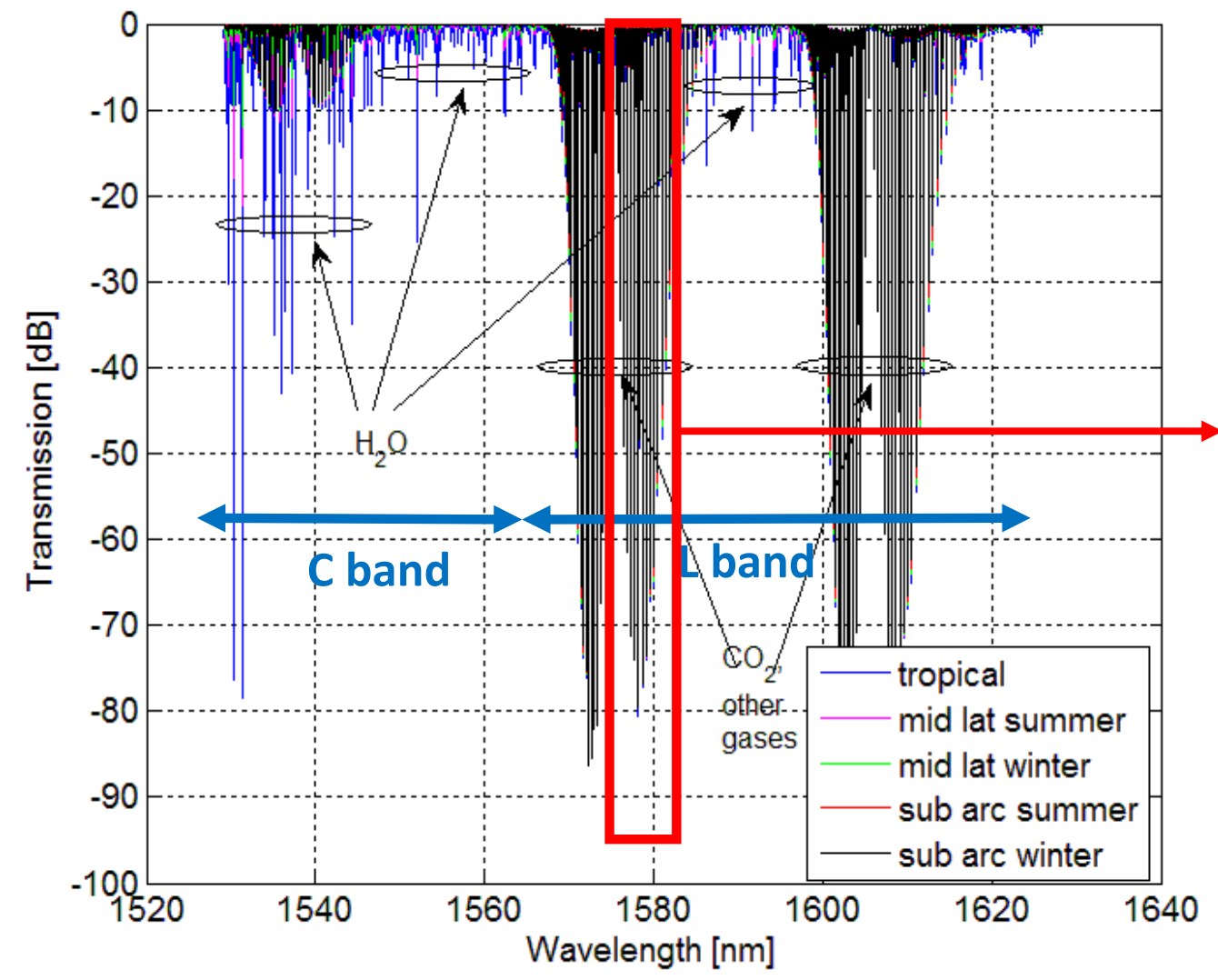

Zoom 2

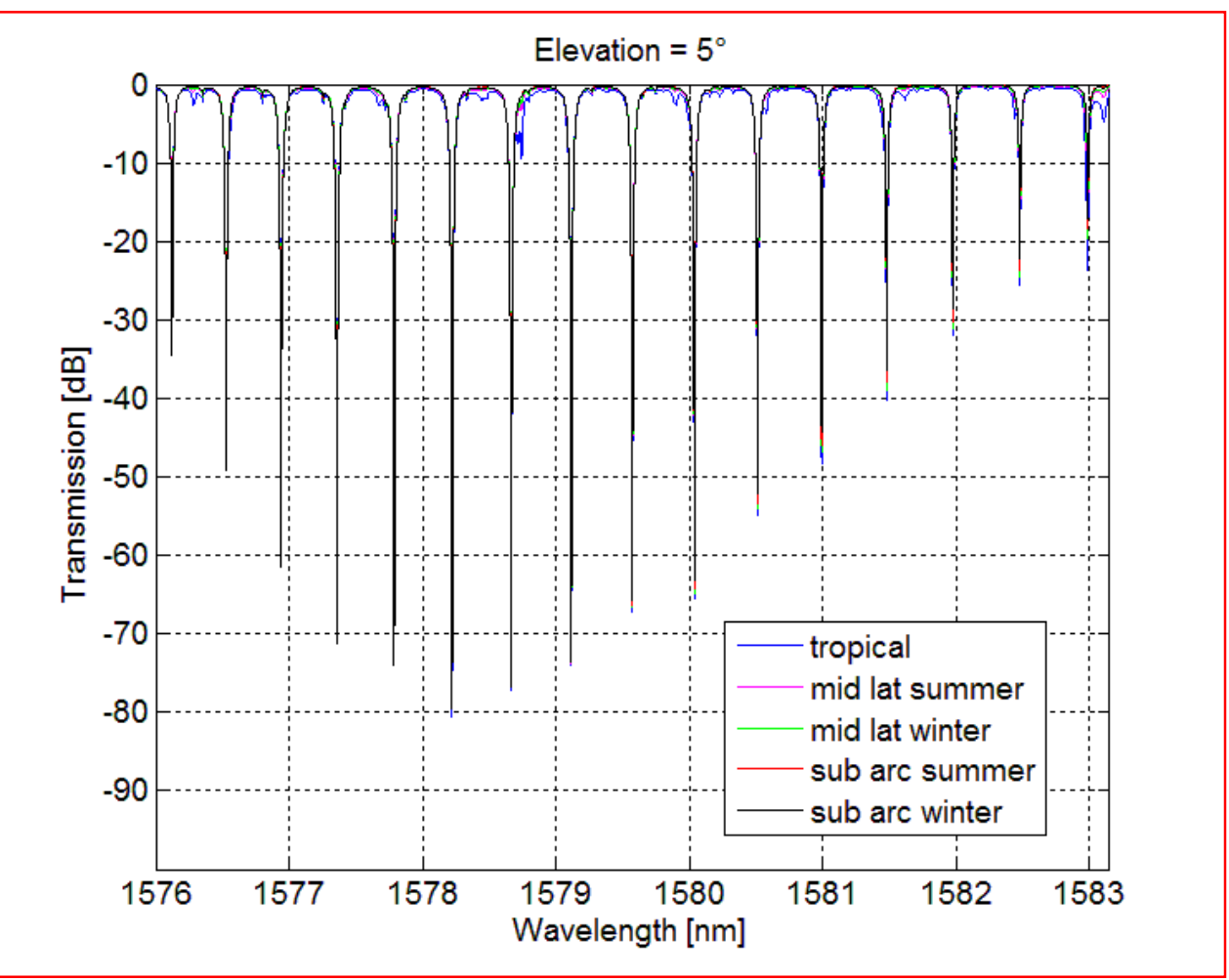


Atmospheric absorption : elevation dependence

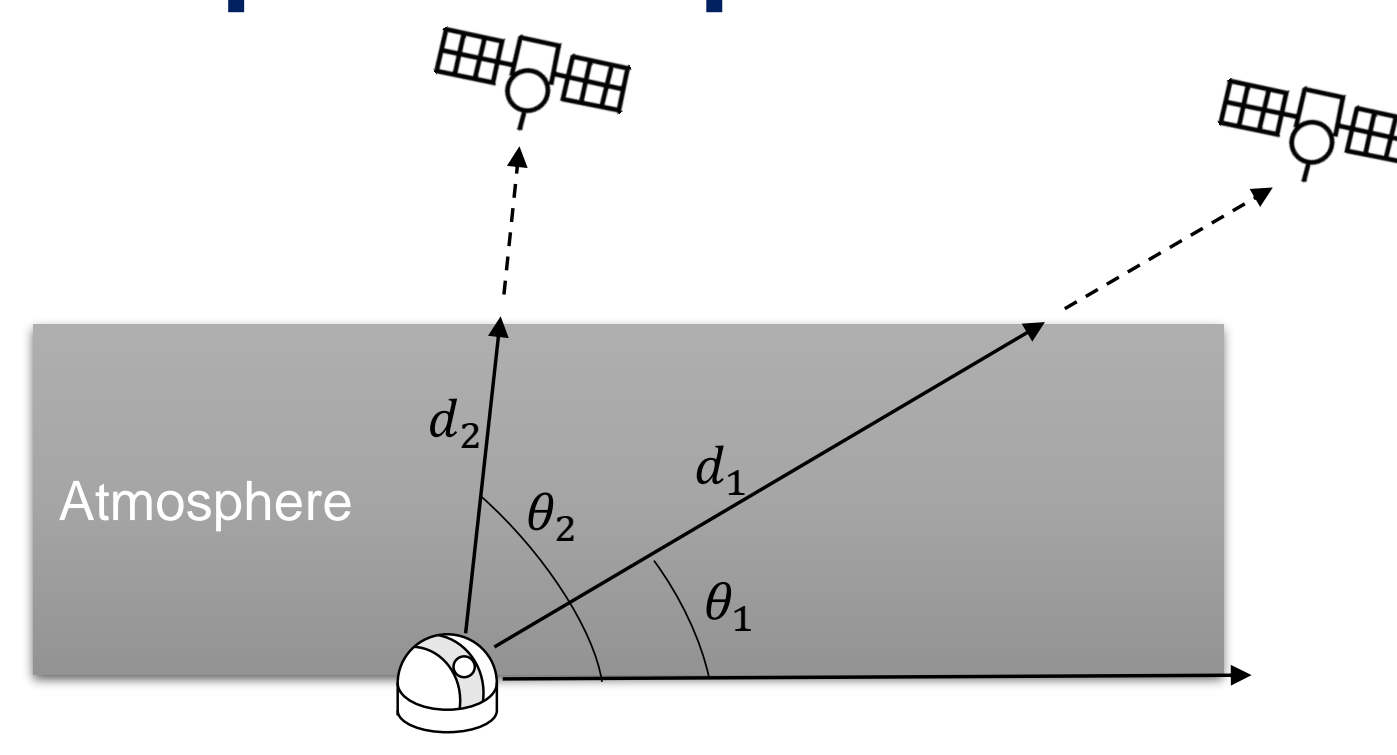

Transmission inv. $\propto$ distance

$$
\theta_{1} \leq \theta_{2} \Rightarrow d_{1} \geq d_{2} \Rightarrow T\left(\theta_{1}\right) \leq T\left(\theta_{2}\right)
$$

The lower the elevation the lower the transmission $=>$ the higher the impairment

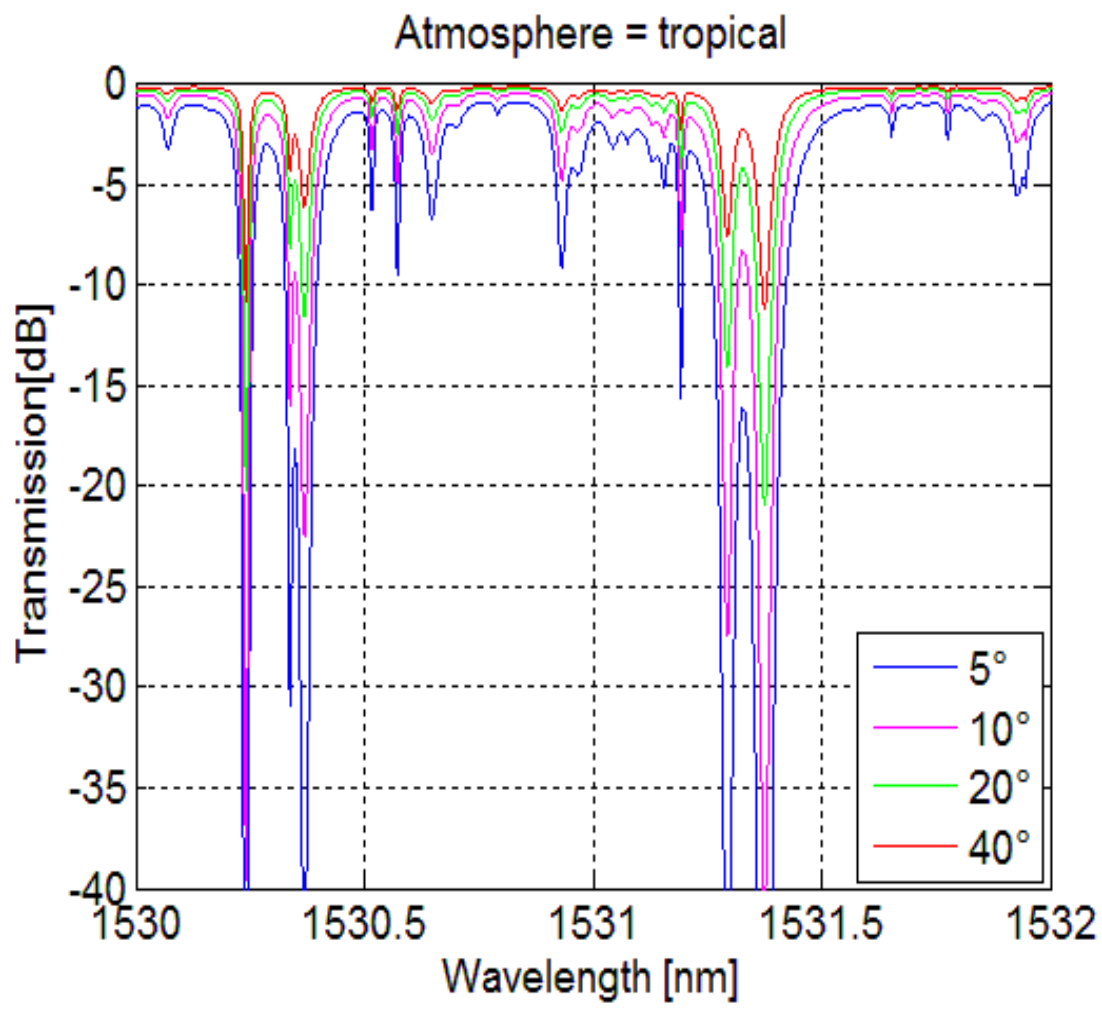




\section{Optical band plan}

Discussions on the suitable band plans in the frame of CCSDS SLS-OPT WG

CCSDS Orange Book for 1550 nm HDR Optical Communications [few Mbps to 100s Gbps] using WDM

Impact of absorption on:

- Acquisition beacon (Pointing Acquisition and Tracking)

- Communication bands WDM wavelengths

ITU grid ITU-T G.694.1:

$$
\mathrm{f}_{\boldsymbol{n}}[\mathbf{T H z}]=193.1 \mathbf{T H z}+n \times \Delta \mathbf{f}[\mathbf{T H z}]
$$

$\Delta f$ : channel spacing $(12.5 \mathrm{GHz}, 25 \mathrm{GHz}, 50 \mathrm{GHz}, 100 \mathrm{GHz} \ldots)$
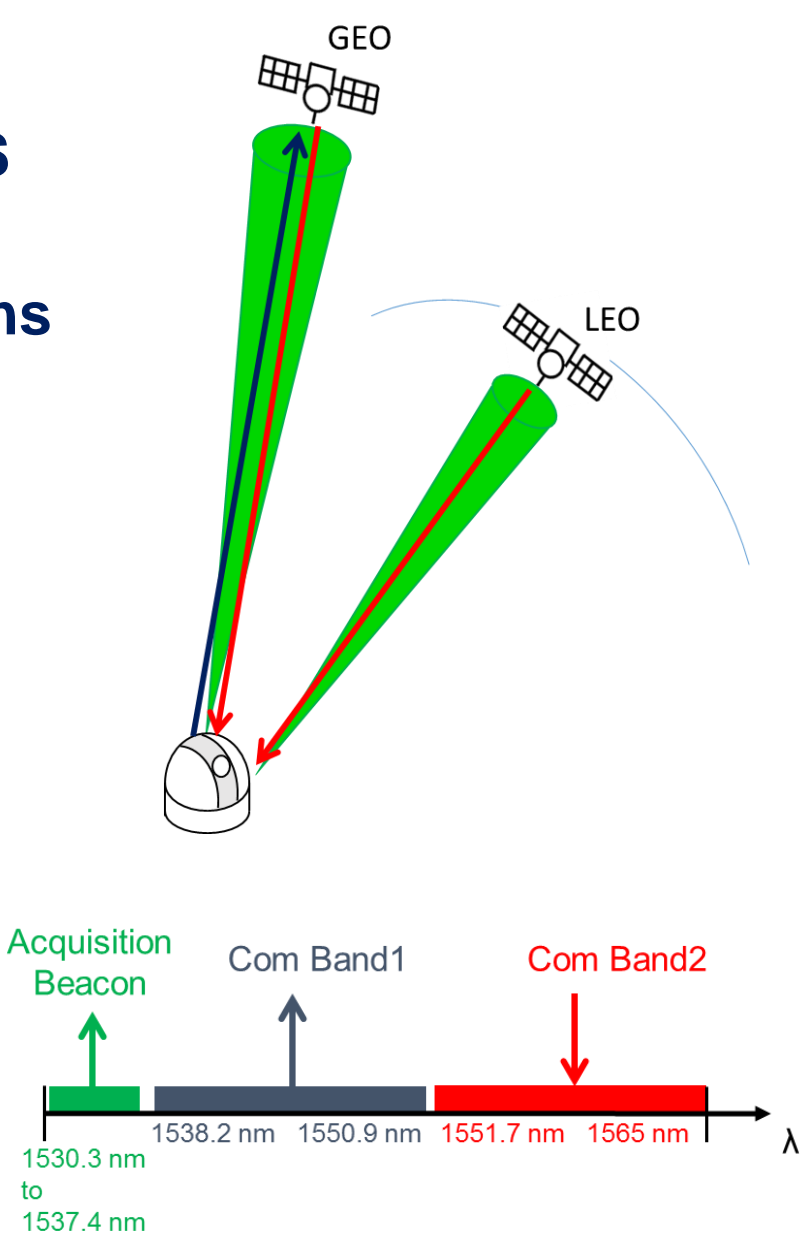


\section{Impact on uplink beacon}

\section{Beacon hypothesis:}

\section{Uplink Beacon}

Line width : narrow $<10 \mathrm{MHz}$

* Central frequency stability vs temperature

$>$ Temperature control: For a variation of $30^{\circ} \mathrm{C}=>+/-9 \mathrm{GHz}$ frequency drift

- Criterion: Maximum absorption line in the selected band

- Orange Book CCSDS-SLS OPT : [1530.3 1537.4] nm

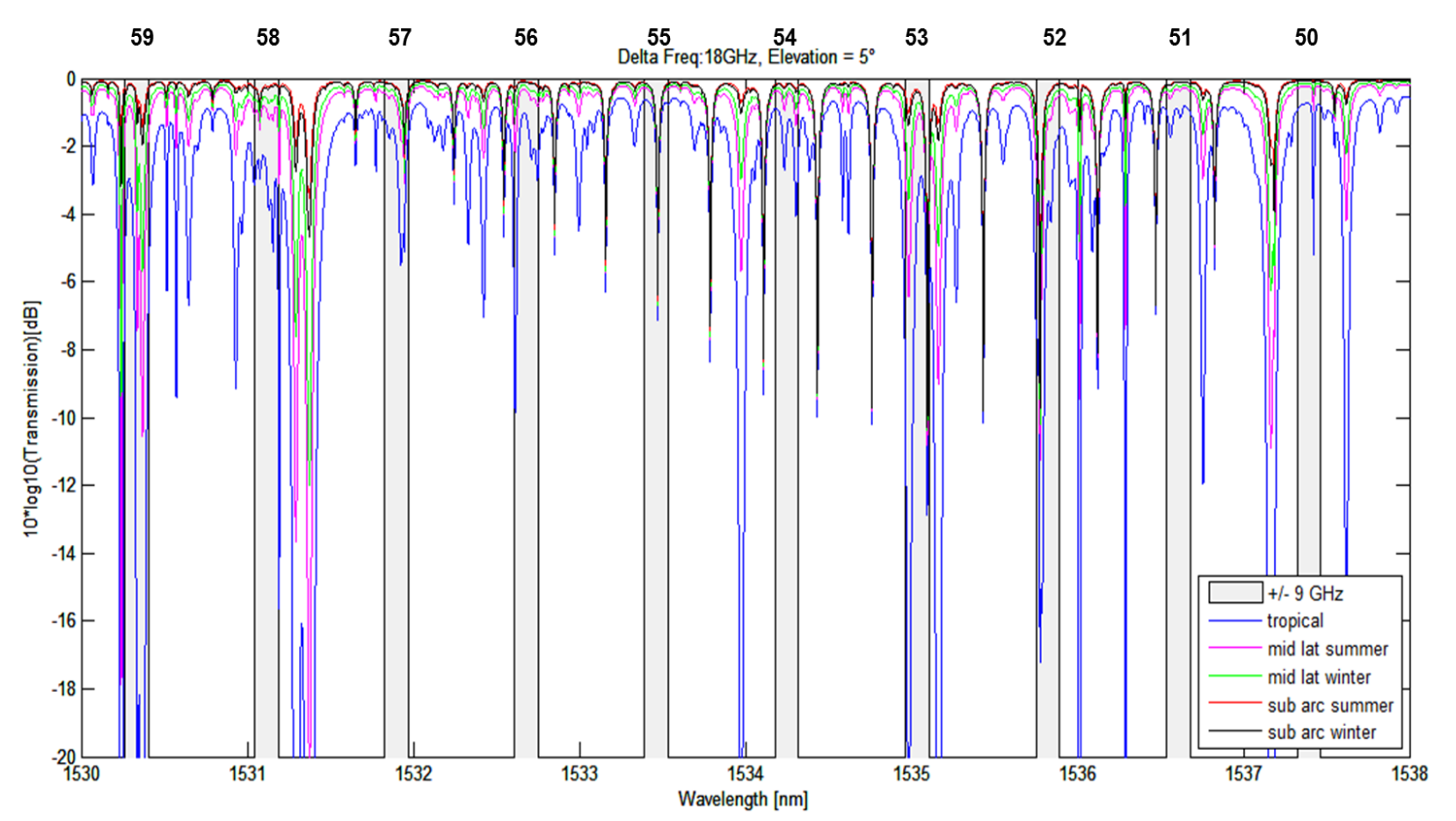




\section{Impact on uplink beacon}

\section{GHz spacing}

- + /-9 GHz integration

- 3dB threshold

- Channel \# (ok, nok, ok if elevation $>=10^{\circ}$ )

\section{E.g. tropical climate :}

- @ $5^{\circ}$ elevation=> only channel 51

- @10 elevation => 3 channels (50, 54 and 57)
$5958575655 \quad 5453525150$
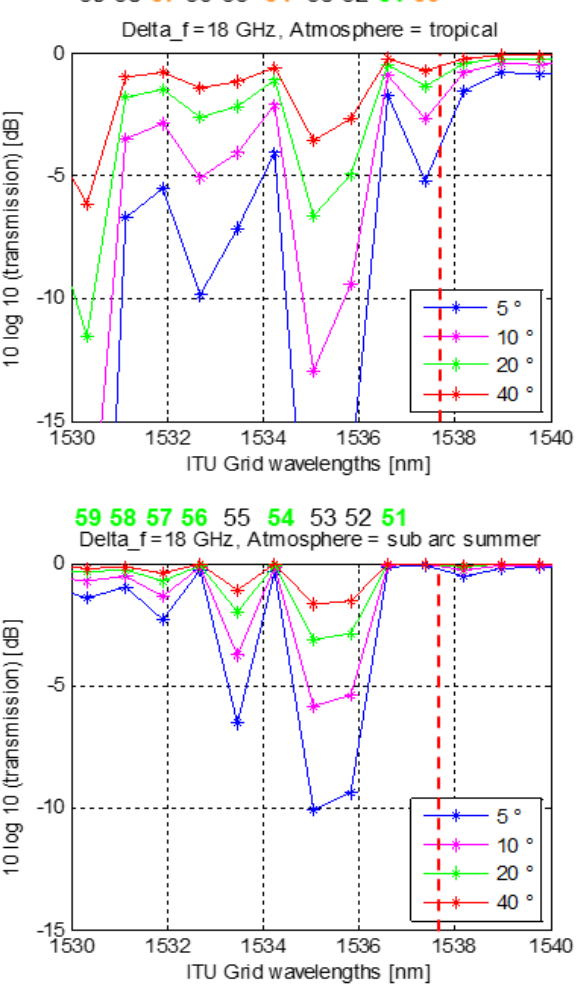

$5958575655 \quad 54 \quad 53525150$

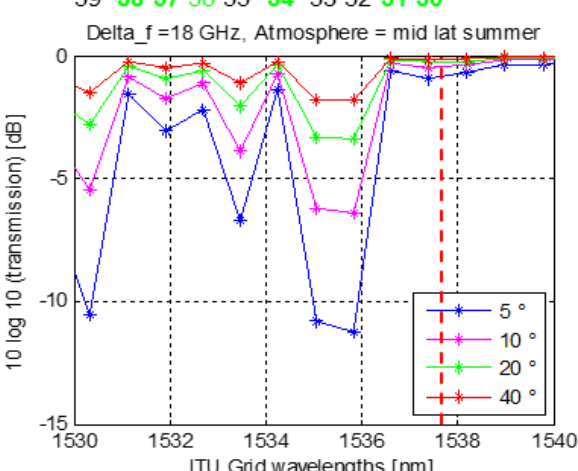

ITU Grid wavelengths [nm]

$59585756 \quad 55 \quad 54535251$

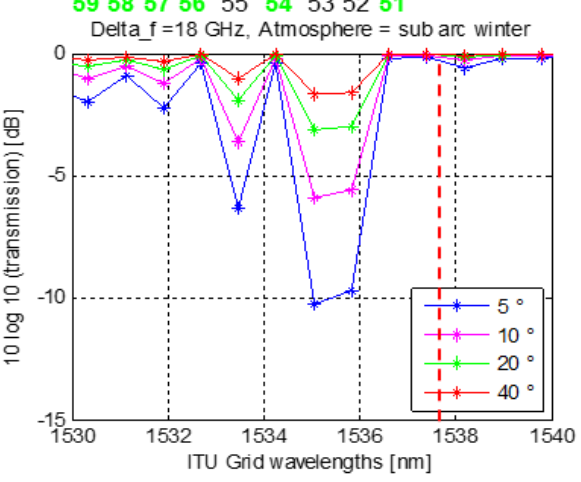

$5958 \quad 5756 \quad 55 \quad 5453525150$ Delta_ $f=18 \mathrm{GHz}$, Atmos phere $=$ mid lat winter

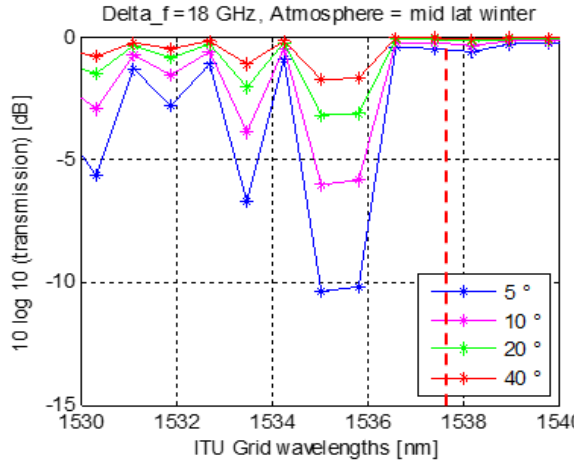




\section{Impact on uplink beacon}

\section{GHz spacing}

- Channels 50, 50.5 ... 59

- $3 d B$ threshold

- Channel \# (ok, nok, ok if elevation $>=10^{\circ}$ )

\section{E.g. tropical climate :}

- @ $5^{\circ}$ elevation=> channels 51 and 51.5

- @10 elevation =>6 channels $(50,54,54.5$, 56.5, 57 and 57.5)

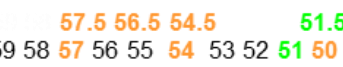
Delta $\mathrm{f}=18 \mathrm{GHz}$, Atmos phere $=$ tropical

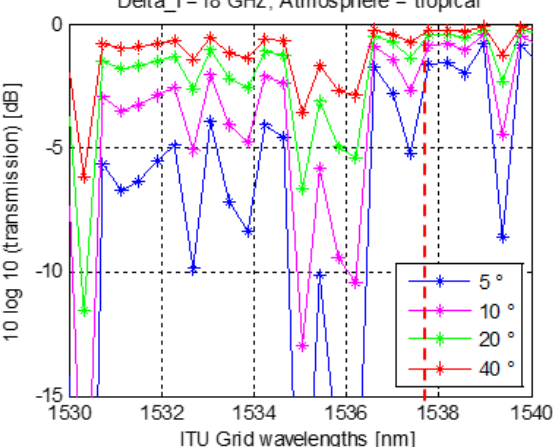

TU Grid wavelengths [nm]

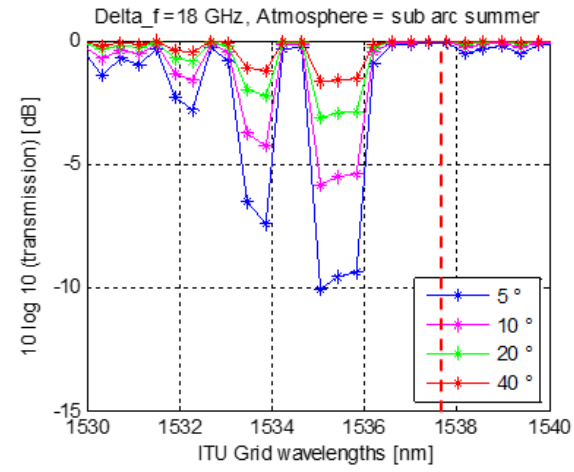

$5958575655 \quad 5453525150$
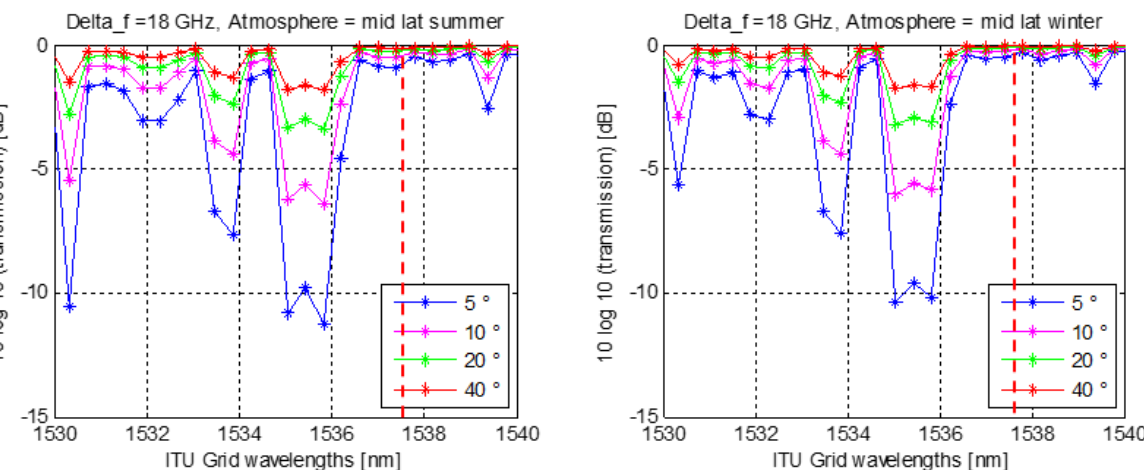

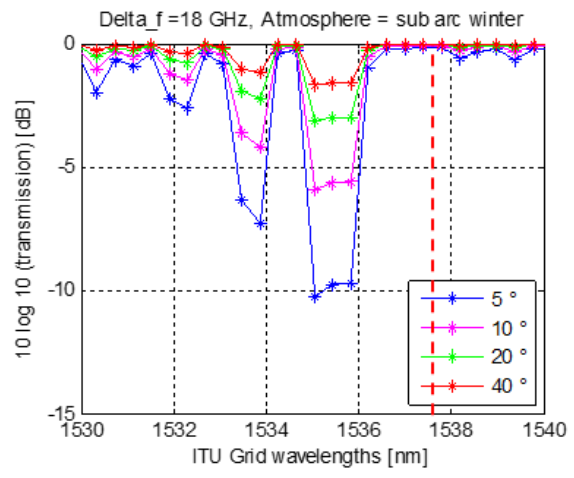




\section{Impact on communications WDM grids}

Impact on the transmission data

- Frequency domain molecular absorption

- 10Gbps communications links

- Integration of $20 \mathrm{GHz}$ around ITU central frequency

- Simulation of end to end optical link performances (BER)
Climate Tropical Elevation $=5$

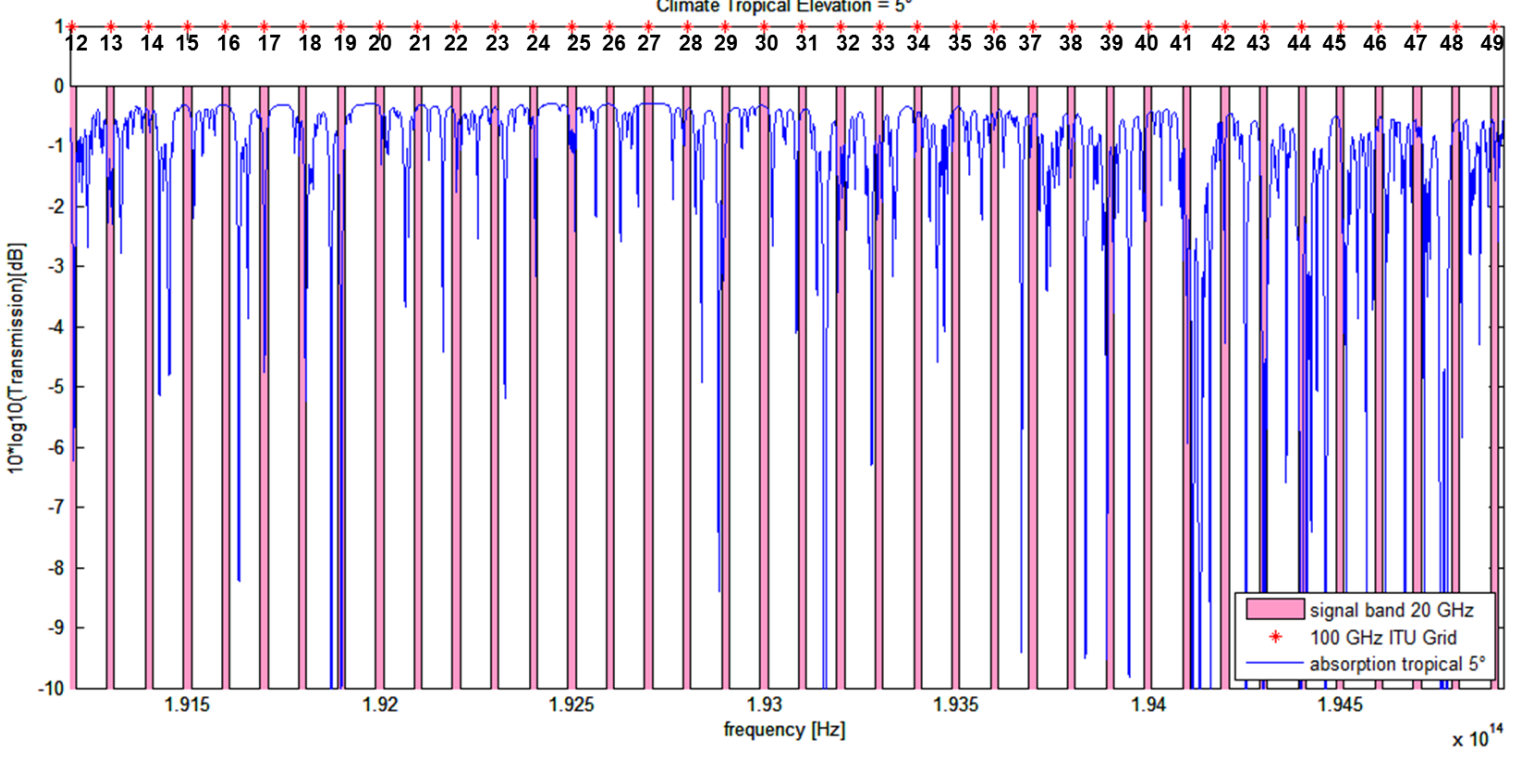




\section{Impact on communications WDM grids}

\section{Simulation of optical} communications end to end link:

- CNES/ISAE-TESA simulator

- NRZ/RZ -OOK (APD or PIN preamplified) and DPSK (PIN preamplified)

- FSO channel includes selective molecular absorption

- Realistic impairments :

- Detection noise

- $\quad$ Band limited receivers

- Laser impairments (relative intensity noise, phase noise)

Simulation of uncoded end to end performance (Bit Error Rate)

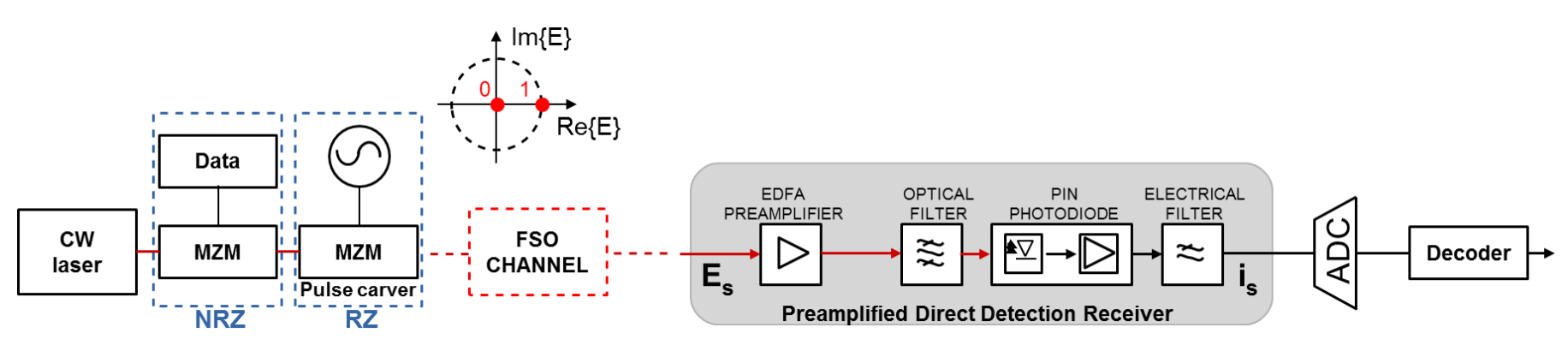

OOK pre-amplified reception

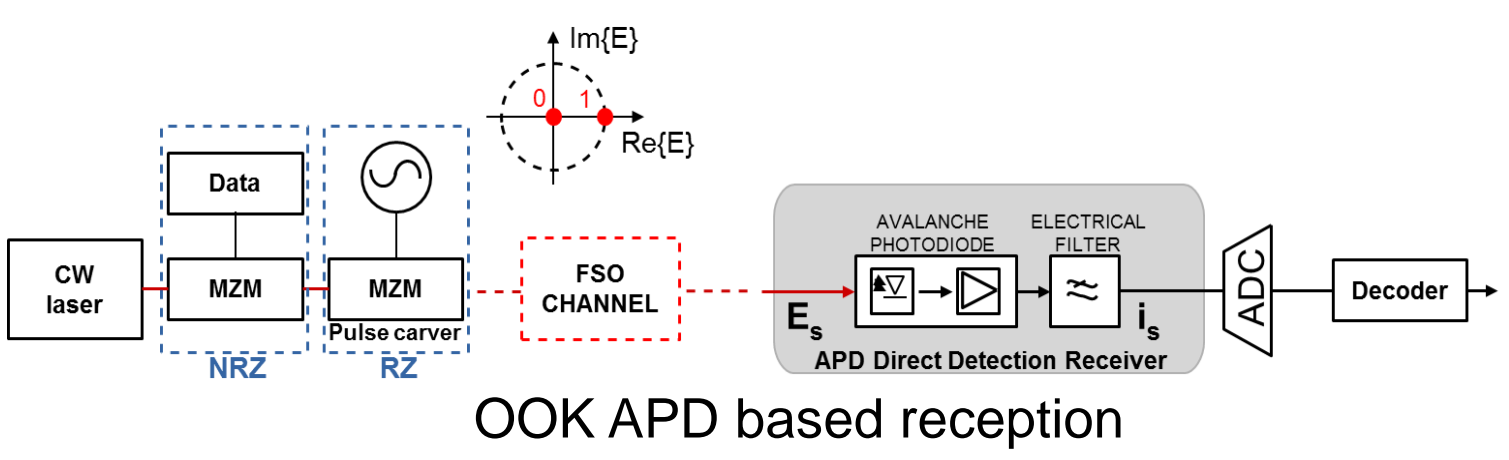




\section{Impact on communications WDM grids}

Application of molecular absorption as a frequency selective filter

$=>$ Power loss and Inter Symbol Interference

Target uncoded Bit error Rate $10^{-4}$

Tropical climate @5 elevation

$=>$ Some channels can have dramatic impact on BER

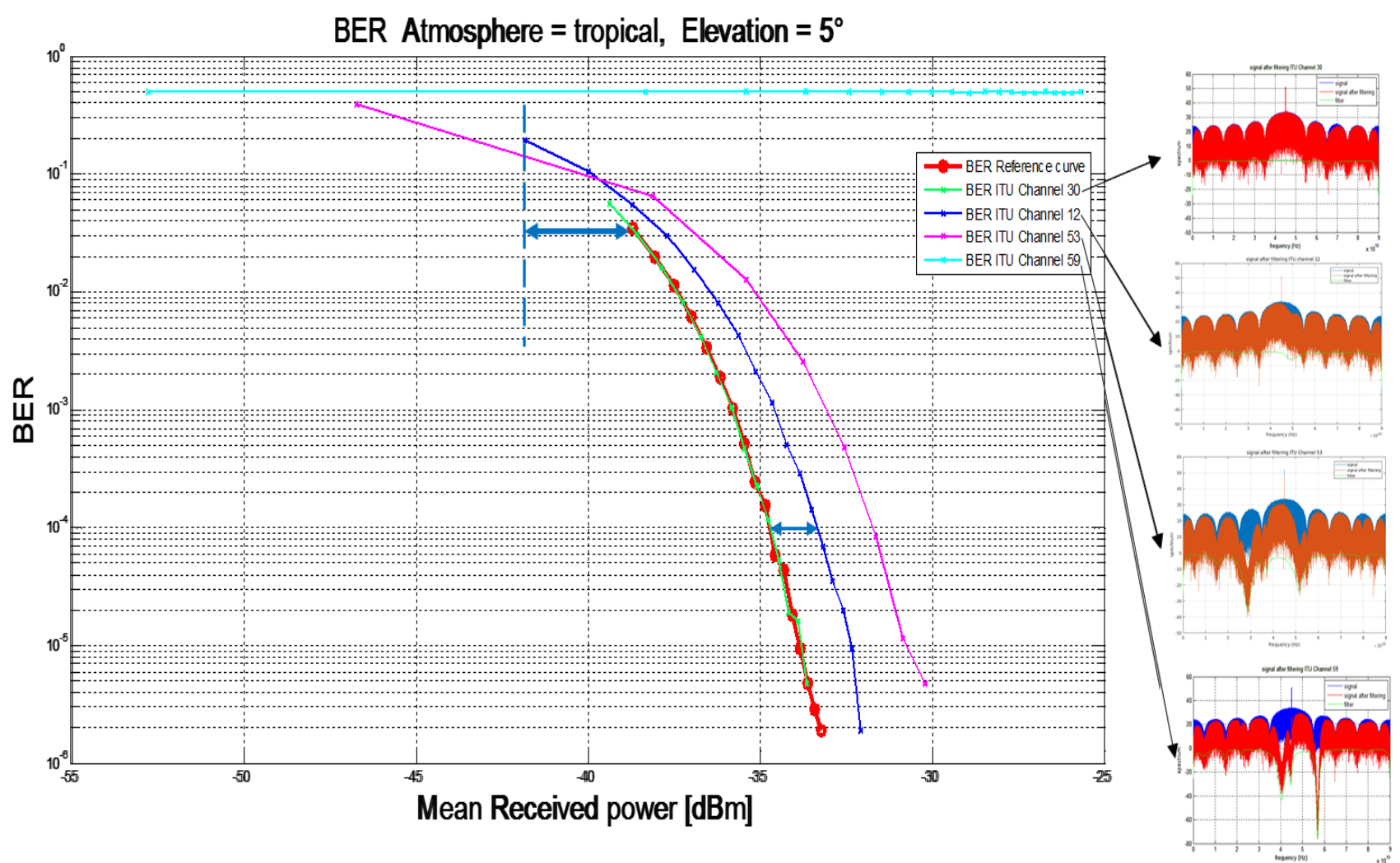




\section{Impact on communications WDM grids}

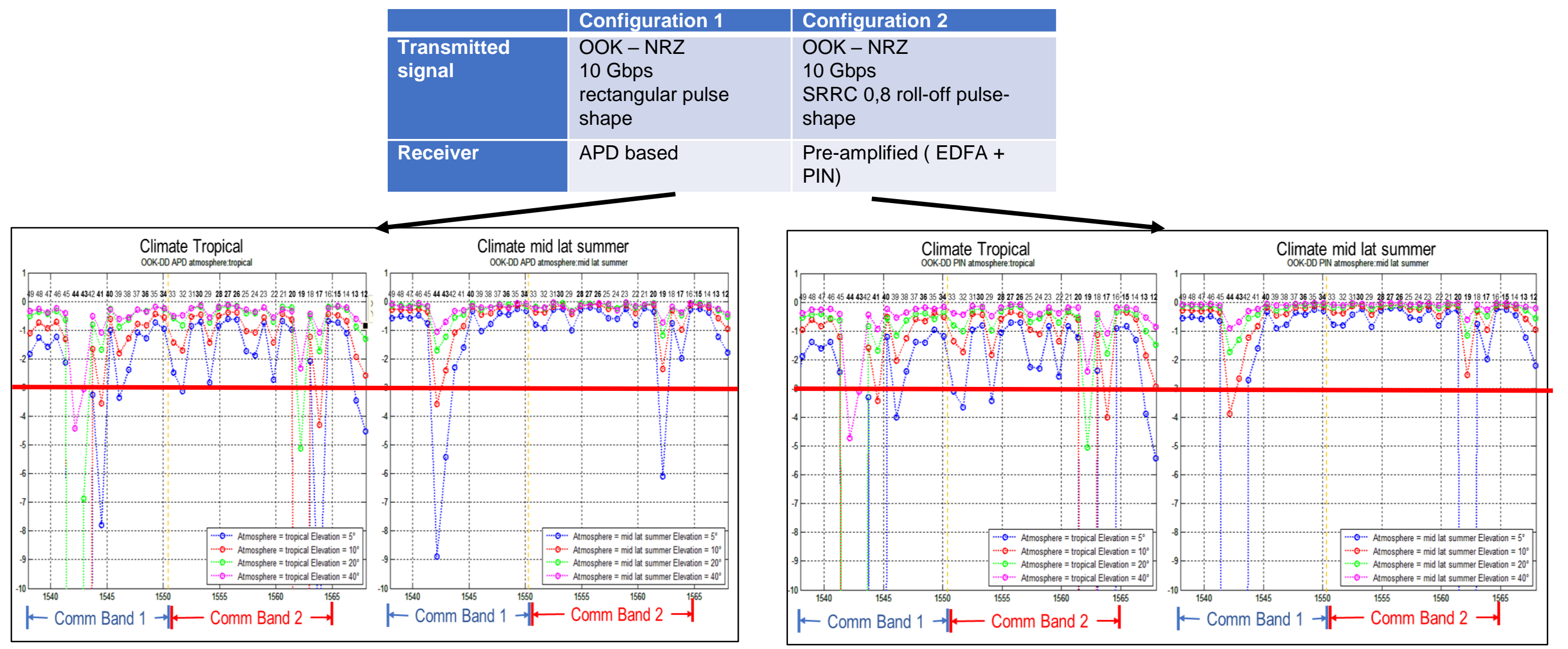




\section{Impact on communications WDM grids}

\section{Number of suitable channels}

- $3 \mathrm{~dB}$ total loss threshold

\begin{tabular}{|l|l|r|r|r|r|r|r|r|r|r|}
\hline & & \multicolumn{3}{|c|}{$5^{\circ}$} & \multicolumn{2}{|c|}{$10^{\circ}$} & \multicolumn{2}{|c|}{$2^{\circ}$} & \multicolumn{2}{|c|}{$40^{\circ}$} \\
\hline & & COM 1 & COM 2 & COM 1 & COM 2 & COM 1 & COM 2 & COM 1 & COM 2 \\
\hline \multirow{3}{*}{ conf 1 } & tropical & 12 & 14 & 14 & 15 & 15 & 16 & 15 & 17 \\
\hline & mid lat sum & 15 & 16 & 16 & 17 & 17 & 17 & 17 & 17 \\
\hline \multirow{2}{*}{ conf 2 } & tropical & mid lat sum & 11 & 13 & 14 & 15 & 15 & 16 & 15 & 17 \\
\hline
\end{tabular}

For worst case scenario (tropical @ $5^{\circ}$ )

- COM band 1 [1538.2;1550.9] nm: 12 channels out of 17

- COM band 2 [1551.7; 1565]: 14 channels out of 17 


\section{Conclusions and perspectives}

\section{For the PAT beacon}

- Several wavelengths could be suitable for uplink beacon on the $100 \mathrm{GHz}$ ITU grid

- More wavelengths can be available by:

- Using the $50 \mathrm{GHz}$ grid

- Ajusting the central wavelength of $<0.1 \mathrm{~nm}$ from the ITU grids

- As multiplexing is not required for beacon, is it compulsory to stick to the ITU grids?

For data communications channels

- When simulating the effect of absorbtion lines on the signal, it appears that more wavelengths are usable than expected in $\mathrm{C}$ band

- Non regular wavelength grids could allow higher number of channels

- Extension of the study to other modulations formats, shaping factors and data rates 